\title{
Hypernovae and their nucleosynthesis
}

\author{
Ken'ichi Nomoto ${ }^{1}$, Keiichi Maeda ${ }^{1}$, Hideyuki Umeda ${ }^{1}$, Takuya Ohkubo ${ }^{1}$, \\ Jingsong Deng ${ }^{1}$, and Paolo Mazzali ${ }^{2}$ \\ ${ }^{1}$ Department of Astronomy, School of Science, University of Tokyo, \\ 7-3-1 Hongo, Bunkyo-ku, Tokyo 113-0033, Japan \\ ${ }^{2}$ Osservatorio Astronomico, Via G.B. Tiepolo 11, I-34131 Trieste, Italia
}

\begin{abstract}
.
We review the characteristics of nucleosynthesis in 'hypernovae', i.e., corecollapse supernovae with very large explosion energies ( $\left.\gtrsim 10^{52} \mathrm{ergs}\right)$. The hypernova yields show the following characteristics: $(i)$ the mass ratio between the complete and incomplete Si burning regions is larger in hypernovae than normal supernovae. As a result, higher energy explosions tend to produce larger [ $\mathrm{Zn}$, $\mathrm{Co}, \mathrm{V}) / \mathrm{Fe}]$ and smaller $[(\mathrm{Mn}, \mathrm{Cr}) / \mathrm{Fe}]$, which could explain the trend observed in very metal-poor stars; (ii) because of enhanced $\alpha$-rich freeze-out, ${ }^{44} \mathrm{Ca},{ }^{48} \mathrm{Ti}$, and ${ }^{64} \mathrm{Zn}$ are produced more abundantly than in normal supernovae. The large $[(\mathrm{Ti}, \mathrm{Zn}) / \mathrm{Fe}]$ ratios observed in very metal poor stars strongly suggest a significant contribution of hypernovae; and (iii) oxygen burning takes place in more extended regions in hypernovae to synthesize a larger amount of $\mathrm{Si}, \mathrm{S}, \mathrm{Ar}$, and $\mathrm{Ca}$ ('Si'), which makes the 'Si'/O ratio larger. The abundance pattern of the starburst galaxy M 82 may be attributed to hypernova explosions. We thus suggest that hypernovae make important contribution to the early Galactic (and cosmic) chemical evolution.
\end{abstract}

\section{Introduction}

One of the most interesting recent developments in the study of supernovae (SNe) is the discovery of some very energetic supernovae (SNe), whose kinetic energy (KE) exceeds $10^{52} \mathrm{erg}$, about 10 times the KE of normal core-collapse SNe (hereafter $E_{51}=E 10^{-51} \mathrm{erg}$ ). Type Ic supernova (SN Ic) $1998 \mathrm{bw}$ was probably linked to GRB 980425 (Galama et al. 1998), thus establishing for the first time a connection between gamma-ray bursts (GRBs) and the well-studied phenomenon of core-collapse SNe. However, SN 1998bw was exceptional for a SN Ic: it was as luminous at peak as a SN Ia, indicating that it synthesized $\sim 0.5 \mathrm{M}_{\odot}$ of ${ }^{56} \mathrm{Ni}$, and its $\mathrm{KE}$ was estimated at $E \simeq 3 \times 10^{52} \mathrm{erg}$ (Iwamoto et al. 1998; Woosley et al. 1999). Because of its large $\mathrm{KE}, \mathrm{SN} 1998 \mathrm{bw}$ was called a 'hypernova'.

Subsequently, other 'hypernovae' of Type Ic have been discovered or recognized, such as SN 1997ef (Iwamoto et al. 2000; Mazzali, Iwamoto \& Nomoto 2000), SN 1997dq (Matheson et al. 2001), SN 1999as (Knop et al. 1999; Hatano et al. 2001), and SN 2002ap (Mazzali et al. 2002). Other possible hypernovae, although of TypeIIn, were SNe 1997cy (Germany et al. 2000; Turatto et al. 2000), and 1999E (Rigon et al. 2002). Figure 1 shows the near-maximum spectra and the absolute $V$-light curves of Type Ic hypernovae. These hypernovae span a 

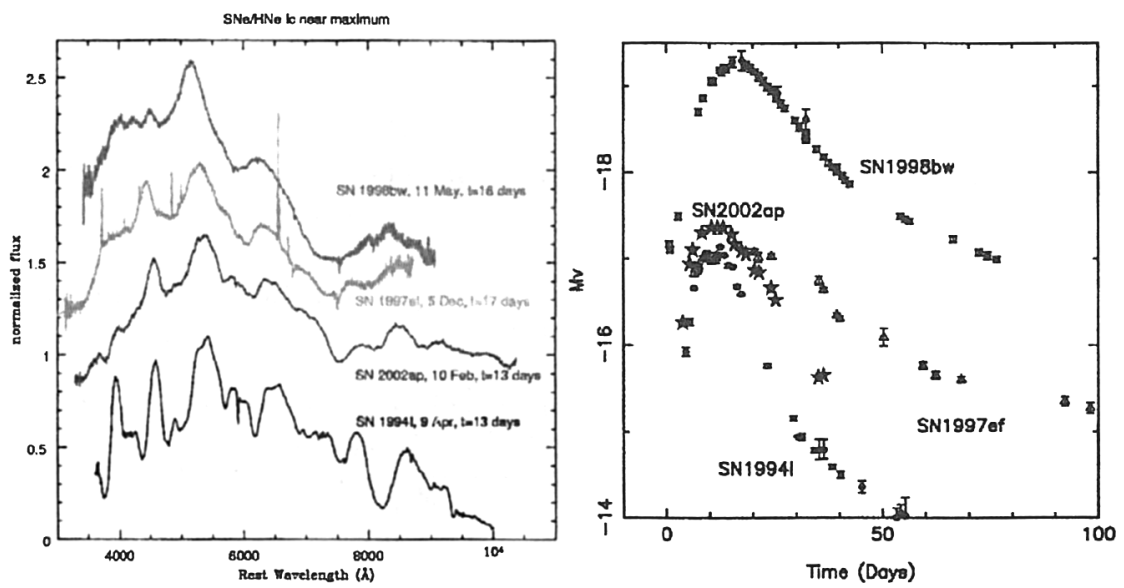

Figure 1. Left: The near-maximum spectra of Type Ic SNe and hypernovae: SNe 1998bw, 1997ef, 2002ap, and 1994I. Right: The observed $V$-band light curves of SN 1998bw (open circles), SN 1997ef (open triangles), SN 2002ap (stars), and SN $1994 \mathrm{I}$ (filled circles) (Mazzali et al. 2002).

wide range of properties, although they all appear to be highly energetic compared to normal core-collapse SNe. SN 1999as is the most luminous supernova ever discovered, reaching a peak magnitude $M_{V}<-21.5$, while the brightness of SN 2002ap appears to be similar to that of normal core-collapse SNe.

In the following sections, we summarize the properties of these hypernovae as derived from optical light curves and spectra. We then show that nucleosynthesis in hypernovae is quite distinct from the case of ordinary supernovae, thus making a unique contribution to galactic chemical evolution.

\section{Hypernova branch and faint supernova branch}

Figure 2 shows $E$ and the mass of ${ }^{56} \mathrm{Ni}$ ejected $-M\left({ }^{56} \mathrm{Ni}\right)$ - as a function of the main-sequence mass $M_{\mathrm{ms}}$ of the progenitor star obtained from fitting the optical light curves and spectra. The estimated masses are $M_{\mathrm{ms}} \gtrsim 60 \mathrm{M}_{\odot}$ for SN1999as, $\sim 40 \mathrm{M}_{\odot}$ for SN1998bw, $\sim 35 \mathrm{M}_{\odot}$ for SN1997ef, and $\sim 20-25 \mathrm{M}_{\odot}$ for SN2002ap. These mass estimates place hypernovae at the high-mass end of SN progenitors, as they are consistently larger than the mass of the progenitors of normal corecollapse $\mathrm{SNe}\left(\sim 10-20 \mathrm{M}_{\odot}\right)$. Our analysis of these objects suggests that the $\mathrm{KE}$ may be related to $M_{\mathrm{ms}} . M\left({ }^{56} \mathrm{Ni}\right)$ also appears to increase with increasing $M_{\mathrm{ms}}$, which is important to know for the study of the chemical evolution of galaxies.

In contrast, SN II 1997D and SN 1999br were very faint SNe with very low KE (Turatto et al. 1998; Hamuy 2002; Zampieri et al. 2002). In Figure 2, therefore, we propose that SNe from stars with $M_{\mathrm{ms}} \gtrsim 20-25 \mathrm{M}_{\odot}$ have different $E$ and $M\left({ }^{56} \mathrm{Ni}\right)$, with a bright, energetic 'hypernova branch' at one extreme and a faint, low-energy SN branch at the other. For the faint SNe, the explosion energy was so small that most ${ }^{56} \mathrm{Ni}$ fell back onto the compact remnant. Thus the faint SN branch may become a 'failed' SN branch at larger $M_{\mathrm{ms}}$. Between the two branches, there may be a variety of SNe (Hamuy 2002). This trend 

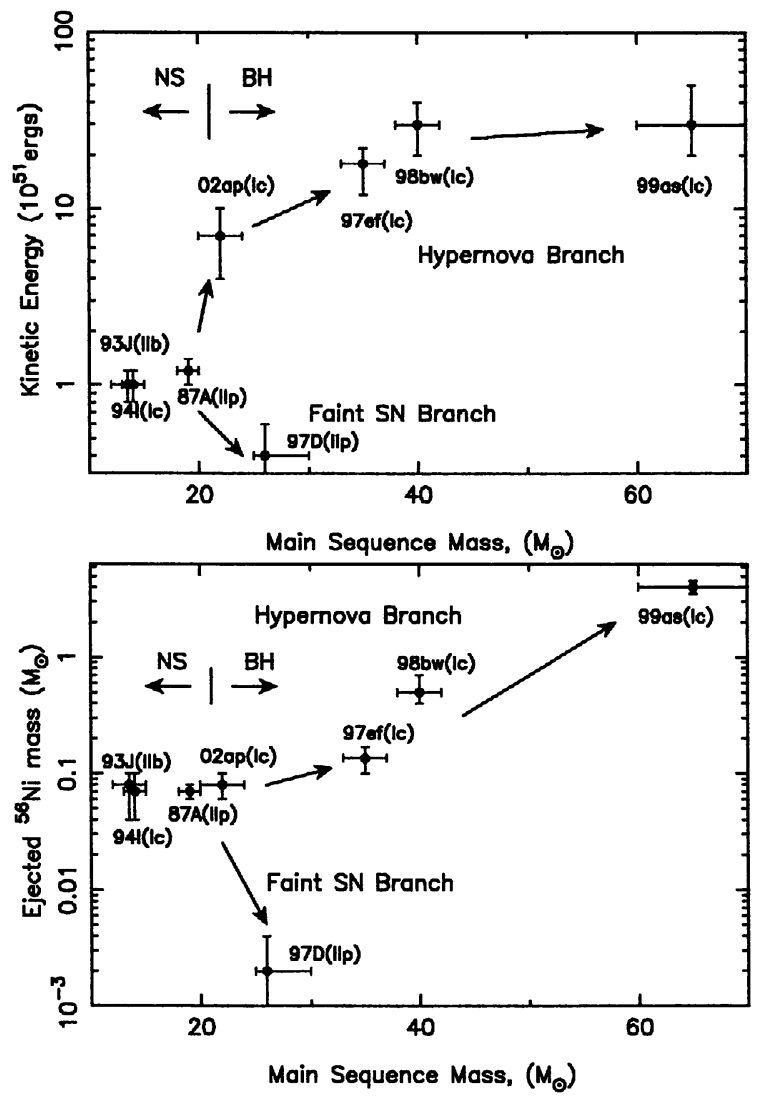

$\therefore$ Figure 2. The explosion energy and the ejected ${ }^{56} \mathrm{Ni}$ mass as a function of the main sequence mass of the progenitors for several supernovae/hypernovae.

might be interpreted as follows. Stars with $M_{\mathrm{ms}} \lesssim 20-25 \mathrm{M}_{\odot}$ form a neutron star (NS), producing $\sim 0.08 \pm 0.03 \mathrm{M}_{\odot}{ }^{56} \mathrm{Ni}$ as in SNe 1993J, 1994I, and 1987A (SN 1987A may be a borderline case between the neutron star and black hole formation). Stars with $M_{\mathrm{ms}} \gtrsim 20-25 \mathrm{M}_{\odot}$ form a black hole (BH); whether they become hypernovae or faint SNe may depend on the angular momentum in the collapsing core, which in turn depends on the stellar winds, metallicity, magnetic fields, and binarity. Hypernovae might have rapidly rotating cores owing possibly to the spiraling-in of a companion star in a binary system.

\section{Aspherical hypernova models}

Although all modeling presented above was performed assuming spherical symmetry, the data show evidence for significant deviations from sphericity. In particular, some polarization was detected in both SNe 1998bw (Iwamoto et al. 1998; Patat et al. 2001) and 2002ap (Kawabata et al. 2002; Leonard et al. 2002; Wang et al. 2002). Furthermore, in the case of SN 1998bw, the late 
time spectra showed peculiar nebular line profiles, where the O I] $6300 \AA$ line is significantly narrower than the Fe II] blend near $5200 \AA$ (Patat et al. 2001).

In spherically symmetric models this is not expected, as $\mathrm{O}$ should always be located above $\mathrm{Fe}$ in velocity space. Moreover, the O I] line declines more slowly than the Fe II] ones, possibly signalling deposition of $\gamma$-rays in a slowly-moving, O-dominated region (Mazzali et al. 2001). Another peculiarity is observed in SN 1997ef, where the photosphere persists to advanced epochs, showing line absorption at velocities of $\sim 2000 \mathrm{~km} \mathrm{~s}^{-1}$, which is well below the expected position of the mass-cut in spherically symmetric models (Mazzali et al. 2000). Finally, all three hypernovae show a late decline of the light curve at epochs of a few months.

Maeda et al. (2002) calculated the nucleosynthesis in aspherical explosions. In such a model, ${ }^{56} \mathrm{Ni}$ is synthesized preferentially along the polar axis, where the $\mathrm{KE}$ is larger, while a lot of unburned material, dominated by $\mathrm{O}$, is left at low velocity in the equatorial region, where burning is much less efficient. A model where the ratio of the polar-to-equatorial kinetic energy is about 8:1 yields an asymmetric explosion whose properties at late times are consistent with the observed lines of SN $1998 \mathrm{bw}$ if it is viewed at an angle of about 15 degrees from the polar axis. At such an angle one might expect that the GRB is weaker than it would be if observed along the jet axis. The actual aspect ratio of the ejecta is much smaller than 8:1, however, as the jet expands laterally, and this may be consistent with the observed polarization.

The asymmetric model has a smaller total kinetic energy than the corresponding symmetric model, as the $\mathrm{KE}$ away from the line of sight is significantly reduced. The estimate, however, is still large, $E_{51} \simeq 10$. The estimate of $M\left({ }^{56} \mathrm{Ni}\right) \simeq 0.6 \mathrm{M}_{\odot}$ from the nebula spectra does not much depend on the asphericity either.

\section{Nucleosynthesis in hypernova explosions}

In core-collapse supernovae/hypernovae, stellar material undergoes shock heating and subsequent explosive nucleosynthesis. Iron-peak elements are produced in two distinct regions, which are characterized by the peak temperature, $T_{\text {peak }}$, of the shocked material. For $T_{\text {peak }}>5 \times 10^{9} \mathrm{~K}$, material undergoes complete $\mathrm{Si}$ burning whose products include $\mathrm{Co}, \mathrm{Zn}, \mathrm{V}$, and some $\mathrm{Cr}$ after radioactive decays. For $4 \times 10^{9} \mathrm{~K}<T_{\text {peak }}<5 \times 10^{9} \mathrm{~K}$, incomplete Si burning takes place and its after decay products include $\mathrm{Cr}$ and $\mathrm{Mn}$ (e.g., Hashimoto, Nomoto, \& Shigeyama 1989; Thielemann, Nomoto, \& Hashimoto 1996).

The right panel of Figure 3 shows the composition in the ejecta of a $25 \mathrm{M}_{\odot}$ hypernova model $\left(E_{51}=10\right)$. The nucleosynthesis in a normal $25 \mathrm{M}_{\odot} \mathrm{SN}$ model $\left(E_{51}=1\right)$ is also shown for comparison in the left panel of Figure 3 .

We note the following characteristics of nucleosynthesis with very large explosion energies (Nomoto et al. 2001a,b):

(i) Both complete and incomplete Si-burning regions shift outward in mass compared with normal supernovae, so that the mass ratio between the complete and incomplete Si-burning regions becomes larger. As a result, higher energy explosions tend to produce larger $[(\mathrm{Zn}, \mathrm{Co}, \mathrm{V}) / \mathrm{Fe}]$ and smaller $[(\mathrm{Mn}, \mathrm{Cr}) / \mathrm{Fe}]$. The elements synthesized in this region such as ${ }^{56} \mathrm{Ni},{ }^{59} \mathrm{Cu},{ }^{63} \mathrm{Zn}$, and ${ }^{64} \mathrm{Ge}$ 

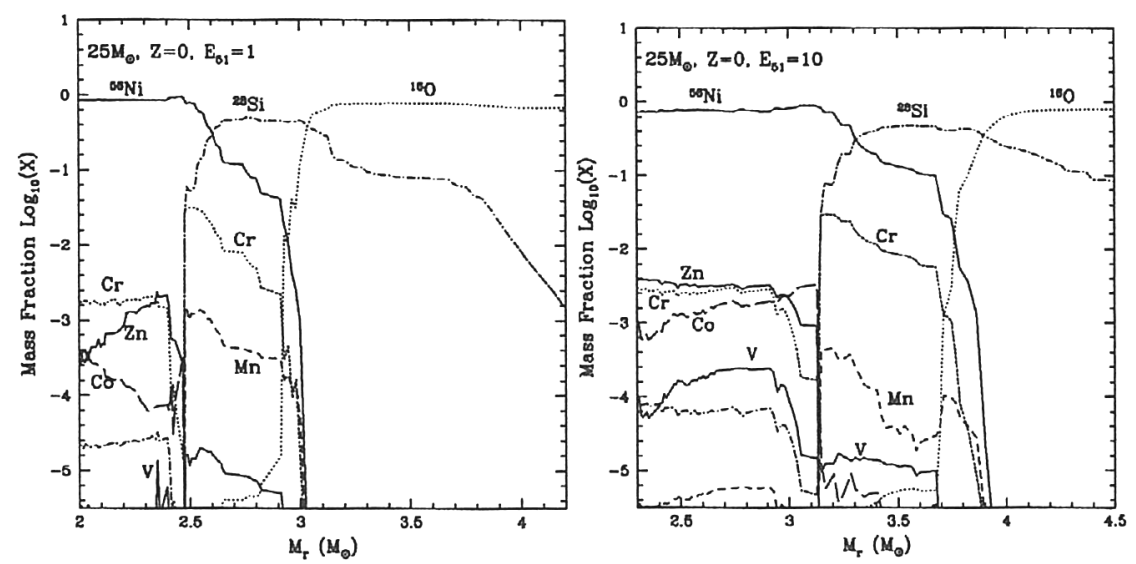

Figure 3. Abundance distribution plotted against the enclosed mass $M_{r}$ after the explosion of Pop III $25 \mathrm{M}_{\odot}$ stars with $E_{51}=1$ (left) and $E_{51}=10$ (right) (Umeda \& Nomoto 2002).

(which decay into ${ }^{56} \mathrm{Fe},{ }^{59} \mathrm{Co},{ }^{63} \mathrm{Cu}$, and ${ }^{64} \mathrm{Zn}$, respectively) are ejected more abundantly than in normal supernovae.

(ii) In the complete Si-burning region of hypernovae, elements produced by $\alpha$-rich freezeout are enhanced because nucleosynthesis proceeds at lower densities (i.e., higher entropy) and thus a larger amount of ${ }^{4} \mathrm{He}$ is left. Hence, elements synthesized through capturing of $\alpha$-particles, such as ${ }^{44} \mathrm{Ti},{ }^{48} \mathrm{Cr}$, and ${ }^{64} \mathrm{Ge}$ (decaying into ${ }^{44} \mathrm{Ca},{ }^{48} \mathrm{Ti}$, and ${ }^{64} \mathrm{Zn}$, respectively) are more abundant.

(iii) Oxygen burning takes place in more extended regions for the larger $\mathrm{KE}$. Then more $\mathrm{O}, \mathrm{C}, \mathrm{Al}$ are burned to produce a larger amount of burning products such as $\mathrm{Si}, \mathrm{S}$, and $\mathrm{Ar}$. Therefore, hypernova nucleosynthesis is characterized by large abundance ratios of $[\mathrm{Si} / \mathrm{O}],[\mathrm{S} / \mathrm{O}],[\mathrm{Ti} / \mathrm{O}]$, and $[\mathrm{Ca} / \mathrm{O}]$.

\section{Hypernovae and galactic chemical evolution}

Hypernova nucleosynthesis may have made an important contribution to Galactic chemical evolution. In the early galactic epoch when the galaxy was not yet chemically well-mixed, $[\mathrm{Fe} / \mathrm{H}]$ may well be determined by mostly a single SN event (Audouze \& Silk 1995). The formation of metal-poor stars is supposed to be driven by a supernova shock, so that $[\mathrm{Fe} / \mathrm{H}]$ is determined by the ejected Fe mass and the amount of circumstellar hydrogen swept-up by the shock wave (Ryan et al. 1996). Then, hypernovae with larger $E$ are likely to induce the formation of stars with smaller $[\mathrm{Fe} / \mathrm{H}]$, because the mass of interstellar hydrogen swept up by a hypernova is roughly proportional to $E$ (Ryan et al. 1996; Shigeyama \& Tsujimoto 1998) and the ratio of the ejected iron mass to $E$ is smaller for hypernovae than for normal supernovae.

\subsection{Zn, Co, $\mathrm{Mn}, \mathrm{Cr}$}

The observed abundances of metal-poor halo stars show quite interesting pattern. There are significant differences between the abundance patterns in the 

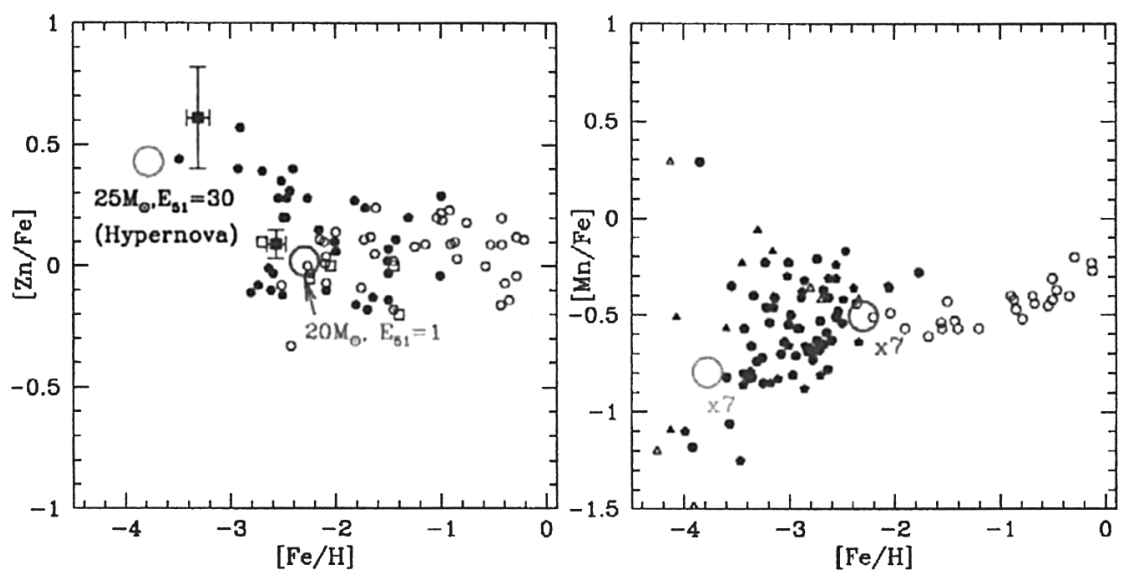

Figure 4. Observed abundance ratios of $[\mathrm{Zn} / \mathrm{Fe}]$ and $[\mathrm{Mn} / \mathrm{Fe}]$ and the theoretical abundance patterns for a normal SN II $\left(20 M_{\odot}, E_{51}=1\right)$ and a hypernova $\left(25 M_{\odot}, E_{51}=30\right)$ models (Umeda \& Nomoto 2002$)$.

iron-peak elements below and above $[\mathrm{Fe} / \mathrm{H}] \simeq-2.5--3:(i)$ For $[\mathrm{Fe} / \mathrm{H}] \lesssim-2.5$, the mean values of $[\mathrm{Cr} / \mathrm{Fe}]$ and $[\mathrm{Mn} / \mathrm{Fe}]$ decrease toward smaller metallicity, while [Co/Fe] increases (Figure 4; McWilliam et al. 1995; Ryan et al. 1996); and (ii) $[\mathrm{Zn} / \mathrm{Fe}] \simeq 0$ for $[\mathrm{Fe} / \mathrm{H}] \simeq-3$ to 0 (Sneden et al. 1991 ), while at $[\mathrm{Fe} / \mathrm{H}]<-3.3$, $[\mathrm{Zn} / \mathrm{Fe}]$ increases toward smaller metallicity (Figure 4; Primas et al. 2000; Blake et al. 2001). These trends cannot be explained with the conventional chemical evolution model that uses previous nucleosynthesis yields.

The larger $[(\mathrm{Zn}, \mathrm{Co}) / \mathrm{Fe}]$ and smaller $[(\mathrm{Mn}, \mathrm{Cr}) / \mathrm{Fe}]$ in the supernova ejecta can be realized if the mass ratio between the complete Si burning region and the incomplete $\mathrm{Si}$ burning region is larger, or equivalently if deep material from the complete Si-burning region is ejected by mixing or aspherical effects. This can be realized if: $(i)$ the mass cut between the ejecta and the compact remnant is located at smaller $M_{r}$ (Nakamura et al. 1999); (ii) $E$ is larger to move the outer edge of the complete Si burning region to larger $M_{r}$ (Nakamura et al. 2001); or (iii) asphericity in the explosion is larger.

Among these possibilities, a large explosion energy $E$ enhances $\alpha$-rich freezeout, which results in an increase of the local mass fractions of $\mathrm{Zn}$ and Co, while $\mathrm{Cr}$ and $\mathrm{Mn}$ are not enhanced (Umeda \& Nomoto 2002). Models with $E_{51}=1$ do not produce sufficiently large $[\mathrm{Zn} / \mathrm{Fe}]$. To be compatible with the observations of $[\mathrm{Zn} / \mathrm{Fe}] \simeq 0.5$, the explosion energy must be much larger, i.e., $E_{51} \gtrsim 20$ for $M \gtrsim 20 \mathrm{M}_{\odot}$, i.e., hypernova-like explosions of massive stars $\left(M \gtrsim 25 \mathrm{M}_{\odot}\right)$ with $E_{51}>10$ are responsible for the production of $\mathrm{Zn}$.

In the hypernova models, the overproduction of $\mathrm{Ni}$, as found in the simple 'deep' mass-cut model, can be avoided. Therefore, if hypernovae made significant contributions to the early Galactic chemical evolution, it could explain the large $\mathrm{Zn}$ and $\mathrm{Co}$ abundances and the small $\mathrm{Mn}$ and $\mathrm{Cr}$ abundances observed in very metal-poor stars as seen in Figure 4. 


\subsection{Fe, Ti}

The Fe mass observed in hypernovae show a trend with the progenitor mass, ranging from $\sim 5 \mathrm{M}_{\odot}$ in SN 1999as to $0.07 \mathrm{M}_{\odot}$ in SN 2002ap. Thus [O/Fe] in the ejecta of most hypernovae may be larger than the solar ratio (see Umeda \& Nomoto 2002). The small [O/Fe] observed in some metal-poor stars and galaxies might be the results of SNe from $13-15 \mathrm{M}_{\odot}$ stars (Nomoto et al. 1997) or possibly very massive hypernovae rather than Type Ia supernovae. In contrast, [O/Fe] must be very large in the faint SN branch. Therefore, the scatter of $[\mathrm{O} / \mathrm{Fe}]$ in metal-poor stars might provide constraints on the fraction of these branches (e.g., Argast et al. 2002).

It has been pointed out that $\mathrm{Ti}$ is deficient in Galactic chemical evolution models using supernova yields currently available (e.g., Timmes et al. 1996; Thielemann et al. 1996), especially at $[\mathrm{Fe} / \mathrm{H}] \lesssim-1$ when $\mathrm{SNe}$ Ia have not contributed to the chemical evolution. However, if the contribution from hypernovae to Galactic chemical evolution is relatively large (or supernovae are more energetic than the typical value of $E_{51}=1$ ), this problem could be relaxed. The $\alpha$-rich freezeout is enhanced in hypernovae, so that ${ }^{48} \mathrm{Ti}$ could be ejected more abundantly.

\section{Starburst galaxy $M 82$ and hypernovae}

$\mathrm{X}$-ray emissions from the starburst galaxy M 82 were observed with ASCA and the abundances of several heavy elements were obtained (Tsuru et al. 1997). Tsuru et al. (1997) found that the overall metallicity of M 82 is quite low, i.e., $\mathrm{O} / \mathrm{H}$ and $\mathrm{Fe} / \mathrm{H}$ are only $0.06-0.05$ times solar, while $\mathrm{Si} / \mathrm{H}$ and $\mathrm{S} / \mathrm{H}$ are $\sim 0.40-$ 0.47 times solar. This implies that the abundance ratios are peculiar, i.e., the ratio $\mathrm{O} / \mathrm{Fe}$ is about solar, while the ratios of $\mathrm{Si}$ and $\mathrm{S}$ relative to $\mathrm{O}$ and $\mathrm{Fe}$ are as high as $\sim 6-8$. These ratios are very different from those ratios in SNeII. Compared with normal SNe II, the important characteristic of hypernova nucleosynthesis is the large $\mathrm{Si} / \mathrm{O}, \mathrm{S} / \mathrm{O}$, and $\mathrm{Fe} / \mathrm{O}$ ratios. The good agreement between the hypernova model $\left(E_{51}=30\right)$ and the observed abundances in M 82 is seen in Umeda et al. (2002).

Hypernovae could also produce larger $E$ per oxygen mass than normal $\mathrm{SNe}$ II, as required for M 82 . We therefore suggest that hypernova explosions may make important contributions to the metal enrichment and energy input to the interstellar matter in M82. The age of starburst activity is estimated to be $\lesssim 10^{7} \mathrm{yr}$ (Strickland 2001), which is so young that only massive stars $\left(M>25 \mathrm{M}_{\odot}\right)$ contributed to nucleosynthesis in $\mathrm{M} 82$.

\section{Concluding remarks}

We have shown that signatures of hypernova nucleosynthesis are seen in the abundance patterns in very metal poor stars and the starburst galaxy M 82 . (See also the abundance pattern in X-ray Nova Sco 1994; Israelian et al. 1999; Podsiadlowski et al. 2002). We suggest that hypernovae of massive stars may make important contributions to the Galactic (and cosmic) chemical evolution, especially in the early low metallicity phase. The IMF of Pop III stars might be different from that of Pop I and II stars, and that more massive stars are abundant for Pop III. 
Acknowledgments. This work has been supported in part by the grantin-Aid for Scientific Research (12640233, 14047206, 14540223) of the Ministry of Education, Science, Culture, Sports, and Technology in Japan.

\section{References}

Argast, D., Samland, M., Thielemann, F.-K., Gerhard, O.E. 2002, A\&A 388, 842

Audouze, J., Silk, J. 1995, ApJ (Letters) 451, L49

Blake, L.A.J., Ryan, S.G., Norris, J.E., Beers, T.C. 2001, Nucl. Phys. A. 688, 502

Galama, T.J., Vreeswijk, P.M., van Paradijs, J., et al. 1998, Nature 395, 670

Germany, L.M., Reiss, D.J., Saler, E.M., et al. 2000, ApJ 533, 320

Hamuy, M. 2002, ApJ submitted

Hashimoto, M., Nomoto, K., Shigeyama, T. 1989, A\&A (Letters) 210, L5

Hatano, K., Branch, D., Nomoto, K., et al. 2001, BAAS 198, 3902

Israelian, G., Rebolo, R., Basri, G., et al. 1999, Nature 401, 142

Iwamoto, K., Mazzali, P.A., Nomoto, K., et al. 1998, Nature 395, 672

Iwamoto, K., Nakamura, T., Nomoto, K., et al. 2000, ApJ 534, 660

Kawabata, K.S., Jeffery, D.J., Iye, M., et al. 2002, ApJ (Letters) 580, L39

Knop, R., Aldering, G., Deustua, S., et al. 1999, IAU Circular No. 7128

Leonard, D.C., Filippenko, A.V., Chornock, R., Foley, R.J. 2002, PASP 114, 1333

Maeda, K., Nakamura, T., Nomoto, K., et al. 2002, ApJ 565, 405

Matheson, T., Filippenko, A.V., Li, W., et al. 2001, AJ 121, 1648

Mazzali, P.A., Iwamoto, K., Nomoto, K. 2000, ApJ 545, 407

Mazzali, P.A., Nomoto, K., Patat, F., Maeda, K. 2001, ApJ 559, 1047

Mazzali, P.A., Deng, J., Maeda, K., Nomoto, K., et al. 2002, ApJ (Letters) 572, L61

McWilliam, A., Preston, G.W., Sneden, C., Searle, L. 1995, AJ 109, 2757

Nakamura, T., Umeda, H., Iwamoto, K., Nomoto, K., Hashimoto, M., Hix, R.W., Thielemann, F.-K. 2001, ApJ 555, 880

Nakamura, T., Umeda, H., Nomoto, K., et al. 1999, ApJ 517, 193

Nomoto, K., Hashimoto, M., Tusjimoto, T., et al. 1997, Nucl. Phys. A616, 79c

Nomoto, K., Mazzali, P.A., Nakmura, T., et al. 2001a, in: M. Livio, N. Panagia \& K. Sahu (eds.), Supernovae and Gamma Ray Bursts, STScI Symp. Ser. 13 (Cambridge: CUP), p. 144

Nomoto, K., Maeda, K., Umeda, H., Nakamura, T. 2001b, in: D. Vanbeveren (ed.), The Influence of Binaries on Stellar Populations Studies (Dordrecht: Kluwer), p. 507

Patat, F., Cappellaro, E., Danziger, J., et al. 2001, ApJ 555, 900

Podsiadlowski, Ph., Nomoto, K., Maeda, K., et al. 2002, ApJ 567, 491

Primas, F., Brugamyer, E., Sneden, C., King, J.R., Beers, T.C., Boesgaard, A.M., Deliyannis, C.P. 2000, in: A. Weiss, T.G. Abel \& V. Hill (eds.) The First Stars (Berlin: Springer), p. 51

Rigon, L., Turatto, M., Benetti, S., et al. 2002, MNRAS submitted

Ryan, S.G., Norris, J.E. Beers, T.C. 1996, ApJ 471, 254

Shigeyama. T., Tsujimoto, T. 1998, ApJ (Letters) 507, L135

Sneden, C., Gratton, R.G., Crocker, D.A. 1991, A\&A 246, 354

Strickland, D.K. 2001, in: R. Fusco-Femiano \& F. Matteucci (eds.), Chemical Enrichment of Intracluster and Intergalactic Medium, ASP-CS 253, 387

Thielemann, F.-K., Nomoto, K., Hashimoto, M. 1996, ApJ 460, 408 
Timmes, F.X., Woosley, S.E., Hartmann, D.H., Hoffman, R.D. 1996, ApJ 464, 332

Tsuru, T. G., Awaki, H., Koyama K., Ptak, A. 1997, PASJ 49, 619

Turatto, M., Mazzali, P.A., Young, T.R., Nomoto, K. et al. 1998, ApJ (Letters) 498, L129

Turatto, M., Suzuki, T., Mazzali, P.A., et al. 2000, ApJ (Letters) 534, L57

Umeda, H., Nomoto, K. 2002, ApJ 565, 385

Umeda, H., Nomoto, K., Tsuru, T.G., Matsumoto, H, 2002, ApJ 578, 855

Wang, L., Baade, D., Höflich, P., et al. 2002, ApJ submitted (astro-ph/0206386)

Woosley, S.E., Eastman, R.G., Schmidt, B.P. 1999, ApJ 516, 788

Zampieri, L., Pastorello, A. Turatto, M., et al. 2002, MNRAS submitted

\section{Discussion}

MATTEuCCI: The hypernova nucleosynthesis you just presented must imply a lower $\mathrm{O} / \mathrm{Fe}$ ratio at low metallicities, at variance with observations. Is that correct?

Nомото: The Fe mass observed in hypernovae seems to increase with the progenitor mass, being as small as $0.07 \mathrm{M}_{\odot}$ from a star of $\sim 25 \mathrm{M}_{\odot}$, as observed in SN 2002ap. Thus the $\mathrm{O} / \mathrm{Fe}$ ratio in the ejecta of most hypernovae is larger than the solar ratio and consistent with the observations.

HOEFLICH: Likely, core collapse SNe are aspherical, and all yields come from spherical models. Asphericity gives high entropy. Do you need both high energy and asphericities for nucleosynthesis?

Nомото: Using the aspherical model which is good for SN $1998 \mathrm{bw}$, we still need about $10^{52}$ erg to explain the observed large $\mathrm{Zn} / \mathrm{Fe}$ ratio. If beaming is too strong, the ejected mass of high-entropy products is also too small.

LANGER: To estimate the influence of hypernovae on galactic nucleosynthesis, it may not be sufficient to consider all stars above (say) $25 \mathrm{M}_{\odot}$ producing hypernova output. You showed that there are also very low energy explosions at high mass, and as discoveries are biased towards the energetic ones, the ratio of hypernovae to low energy explosions may be large.

Nомото: The fractions of hypernovae and faint supernovae among massive stars are very uncertain. However, hypernovae tend to contribute to produce stars with small $\mathrm{Fe} / \mathrm{H}$, because the swept-up mass of interstellar matter is larger for a larger energy explosion and thus the resulting $\mathrm{Fe} / \mathrm{H}$ is smaller.

LEITHERER: What would a 'hypernova' remnant look like? The SNR population of M 82 has been observed quite extensively. Does it give clues on the nature of the progenitor?

Nомото: Hypernova explosions are of course much more energetic than ordinary supernovae, thus producing bubbles with higher velocities and entropies. Hypernovae are also expected to produce more aspherical remnants than ordinary SNRs. However, although the abundance distribution is quite aspherical, the density distribution is not so aspherical. Thus it may not be easy to identify the hypernova remnants just from their shapes. 